EPJ Web of Conferences 113,04012 (2016)

DOI: $10.1051 /$ epjconf/201611304012

(C) Owned by the authors, published by EDP Sciences, 2016

\title{
Reactions in the four-nucleon system above breakup threshold
}

\author{
A. Deltuva ${ }^{1, a}$ and A. C. Fonseca ${ }^{2, b}$ \\ ${ }^{1}$ Institute of Theoretical Physics and Astronomy, Vilnius University, A. Goštauto 12, LT-01108 Vilnius, Lithua- \\ nia \\ ${ }^{2}$ Centro de Física Nuclear da Universidade de Lisboa, P-1649-003 Lisboa, Portugal
}

\begin{abstract}
Microscopic calculations of four-body scattering become very challenging in the energy regime above the threshold for four free particles. We consider mixed-isospin four-nucleon reactions initiated by the proton- ${ }^{3} \mathrm{H}$, neutron- ${ }^{3} \mathrm{He}$, or deuteron-deuteron collisions. We solve the Alt, Grassberger, and Sandhas equations for the four-nucleon transition operators in the momentum-space framework. The complex-energy method with special integration weights is applied to deal with the complicated singularities in the kernel of AGS equations. Results for the differential cross section and spin observables in elastic, charge-exchange, transfer, and breakup reactions using realistic potentials are presented.
\end{abstract}

\section{Introduction}

The four-nucleon $(4 \mathrm{~N})$ continuum is an important "theoretical laboratory" for a quantitative test of the two-nucleon $(2 \mathrm{~N})$ and three-nucleon $(3 \mathrm{~N})$ interaction models. However, its theoretical description is technically and computationally highly challenging. Exact four-particle scattering equations have been formulated 50 years ago by Faddeev and Yakubovsky (FY) [1] and by Alt, Grassberger, and Sandhas (AGS) [2,3]. However, their numerical solution using realistic force models only became possible many years later. In the last decade accurate numerical calculations for low-energy nucleon-trinucleon elastic scattering have been performed using both coordinate-space and momentum-space rigorous approaches, namely, the hyperspherical harmonics $(\mathrm{HH})$ expansion method [4, 5], the Faddeev-Yakubovsky (FY) equations [1] for the wave function components [6], and the Alt, Grassberger and Sandhas (AGS) equations [3] for transition operators [7, 8]. The reliability of all these methods was confirmed in a benchmark calculation [9] for neutron- ${ }^{3} \mathrm{H}\left(n-{ }^{3} \mathrm{H}\right)$ and proton $-{ }^{3} \mathrm{He}\left(p-{ }^{3} \mathrm{He}\right)$ elastic scattering observables.

Above the four-body breakup threshold the calculations become even more difficult owing to singularities in the momentum-space or complicated boundary conditions in the coordinate-space framework. In this regime rigorous and realistic results have been obtained so far by only two methodologies. These are the complex-energy method in the framework of momentum-space integral equations [10] and the complex scaling method in the framework of coordinate-space differential equations [11]. Only the former method has been applied to reactions involving the coupled proton $-{ }^{3} \mathrm{H}\left(p-{ }^{3} \mathrm{H}\right)$,

\footnotetext{
a e-mail: arnoldas.deltuva@tfai.vu.lt

be-mail: fonseca@cii.fc.ul.pt
} 
neutron- ${ }^{3} \mathrm{He}\left(n-{ }^{3} \mathrm{He}\right)$ and deuteron-deuteron $(d-d)$ systems. The scattering process in these systems resembles a typical nuclear reaction where elastic, charge exchange, transfer, and breakup reactions take place simultaneously. The present contribution summarizes the achievements in the calculation of $4 N$ reactions initiated by $p+{ }^{3} \mathrm{H}, n+{ }^{3} \mathrm{He}$, or $d+d$ collisions above the breakup threshold.

\section{Theory}

Four-nucleon scattering process is described exactly using the Alt, Grassberger, and Sandhas (AGS) equations [3]. The symmetrized version for the four-particle transition operators $\mathcal{U}_{\beta \alpha}$ is derived in ref. [7], where the nucleons are treated as identical particles in the isospin formalism, i.e.,

$$
\begin{aligned}
& \mathcal{U}_{11}=-\left(G_{0} t G_{0}\right)^{-1} P_{34}-P_{34} U_{1} G_{0} t G_{0} \mathcal{U}_{11}+U_{2} G_{0} t G_{0} \mathcal{U}_{21}, \\
& \mathcal{U}_{21}=\left(G_{0} t G_{0}\right)^{-1}\left(1-P_{34}\right)+\left(1-P_{34}\right) U_{1} G_{0} t G_{0} \mathcal{U}_{11}, \\
& \mathcal{U}_{12}=\left(G_{0} t G_{0}\right)^{-1}-P_{34} U_{1} G_{0} t G_{0} \mathcal{U}_{12}+U_{2} G_{0} t G_{0} \mathcal{U}_{22}, \\
& \mathcal{U}_{22}=\left(1-P_{34}\right) U_{1} G_{0} t G_{0} \mathcal{U}_{12} .
\end{aligned}
$$

Here, $\alpha=1$ corresponds to the $3+1$ partition $(12,3) 4$, whereas $\alpha=2$ corresponds to the $2+2$ partition (12)(34). The free resolvent

$$
G_{0}=\left(Z-H_{0}\right)^{-1}
$$

is taken with a complex energy parameter $Z=E+i \varepsilon$, where $H_{0}$ is the free Hamiltonian $H_{0}$; the operator

$$
t=v+v G_{0} t
$$

is the pair (12) transition matrix resulting from the potential $v$, and

$$
U_{\alpha}=P_{\alpha} G_{0}^{-1}+P_{\alpha} t G_{0} U_{\alpha}
$$

are the $3+1$ or $2+2$ subsystem transition operators. For the four-nucleon system the basis states are antisymmetric under exchange of two particles in the subsystem (12) and, in the $2+2$ partition, also in the subsystem (34). The full antisymmetry is ensured by the permutation operators $P_{a b}$ of particles $a$ and $b$ with $P_{1}=P_{12} P_{23}+P_{13} P_{23}$ and $P_{2}=P_{13} P_{24}$.

The scattering amplitudes for two-cluster reactions at available energy $E=\epsilon_{\alpha}+p_{\alpha}^{2} / 2 \mu_{\alpha}=\epsilon_{\beta}+$ $p_{\beta}^{2} / 2 \mu_{\beta}$ are obtained from the on-shell matrix elements $\left\langle\mathbf{p}_{\beta}\left|T_{\beta \alpha}\right| \mathbf{p}_{\alpha}\right\rangle=S_{\beta \alpha}\left\langle\phi_{\beta}\left|\mathcal{U}_{\beta \alpha}\right| \phi_{\alpha}\right\rangle$ in the limit $\varepsilon \rightarrow+0$. Here $\left|\phi_{\alpha}\right\rangle$ is the Faddeev component of the asymptotic two-cluster state in the channel $\alpha$, characterized by the bound state energy $\epsilon_{\alpha}<0$, the relative momentum $\mathbf{p}_{\alpha}$, and the reduced mass $\mu_{\alpha}$; $S_{\beta \alpha}$ are the symmetrization factors [7]. The AGS equations (1) are solved in the momentum-space partial-wave framework. Two different types of basis states $\left|k_{x} k_{y} k_{z} v\right\rangle_{\alpha}$ with $\alpha=1$ and 2 are employed, where $k_{x}, k_{y}$, and $k_{z}$ denote magnitudes of the Jacobi momenta, and $v$ abbreviates all discrete quantum numbers. The reduced masses associated with Jacobi momenta $k_{x}$ and $k_{y}$ in the partition $\alpha$ are denoted by $\mu_{\alpha x}$ and $\mu_{\alpha y}$, respectively.

In the solution process the integrals are discretized using Gaussian quadrature rules [12] turning Eqs. (1) into a system of linear equations as described in ref. [7]. However, in the limit $\varepsilon \rightarrow+0$ needed for the calculation of the observables the kernel of the AGS equations contains integrable singularities: At $E+i \varepsilon-\epsilon_{\alpha}-k_{z}^{2} / 2 \mu_{\alpha} \rightarrow 0$ the subsystem transition operator in the bound state channel has the pole

$$
G_{0} U_{\alpha} G_{0} \rightarrow \frac{P_{\alpha}\left|\phi_{\alpha}\right\rangle S_{\alpha \alpha}\left\langle\phi_{\alpha}\right| P_{\alpha}}{E+i \varepsilon-\epsilon_{\alpha}-k_{z}^{2} / 2 \mu_{\alpha}} .
$$


At $E+i \varepsilon-\epsilon_{d}-k_{y}^{2} / 2 \mu_{\alpha y}-k_{z}^{2} / 2 \mu_{\alpha} \rightarrow 0$ the two-nucleon transition matrix in the channel with the deuteron quantum numbers for the pair (12) has the pole

$$
t \rightarrow \frac{v\left|\phi_{d}\right\rangle\left\langle\phi_{d}\right| v}{E+i \varepsilon-\epsilon_{d}-k_{y}^{2} / 2 \mu_{\alpha y}-k_{z}^{2} / 2 \mu_{\alpha}}
$$

with $\left|\phi_{d}\right\rangle$ being the pair (12) deuteron wave function and $\epsilon_{d}$ being internal deuteron energy. At $E+i \varepsilon-$ $k_{x}^{2} / 2 \mu_{\alpha x}-k_{y}^{2} / 2 \mu_{\alpha y}-k_{z}^{2} / 2 \mu_{\alpha} \rightarrow 0$ the free resolvent (2) becomes singular as well. The interplay of all these singularities with permutation operators and basis transformations leads to a very complicated singularity structure of the AGS equations.

This difficulty can be formally avoided by performing calculations for a set of finite $\varepsilon>0$ values where the kernel contains no singularities and then extrapolating the results to the $\varepsilon \rightarrow+0$ limit. This idea was proposed in Refs. [13-15] and the point method [13] was suggested for the extrapolation. However, this extrapolation method as well as alternative choices are only precise if $\varepsilon$ is small enough. However, for in that case the kernel of the AGS equations, although formally being non-singular, exhibits a quasi-singular behavior and therefore requires dense grids for the numerical integration. In practical calculations with large number of partial waves necessary for the convergence it is highly desirable to keep the number of integration grid points as small as possible. Thus, a more sophisticated integration method is needed.

An important technical improvement when calculating $\mathcal{U}_{\beta \alpha}$ at finite $\varepsilon$ was introduced in Ref. [10]. The method of special weights for numerical integration involving any of the above-mentioned quasisingularities is used, i.e.,

$$
\int_{a}^{b} \frac{f(x)}{x_{0}^{n}+i y_{0}-x^{n}} d x \approx \sum_{j=1}^{N} f\left(x_{j}\right) w_{j}\left(n, x_{0}, y_{0}, a, b\right) .
$$

The quasi-singular factor $\left(x_{0}^{n}+i y_{0}-x^{n}\right)^{-1}$ is separated and absorbed into the special integration weights $w_{j}\left(n, x_{0}, y_{0}, a, b\right)$. The set of $N$ grid points $\left\{x_{j}\right\}$ where the remaining smooth function $f(x)$ has to be evaluated is chosen the same as for the standard Gaussian quadrature. However, while the standard weights are real [12], the special weights $w_{j}\left(n, x_{0}, y_{0}, a, b\right)$ are complex. They are chosen such that for a set of $N$ spline functions $S_{j}(x)$ the result (7) is exact. The corresponding special weights are

$$
w_{j}\left(n, x_{0}, y_{0}, a, b\right)=\int_{a}^{b} \frac{S_{j}(x)}{x_{0}^{n}+i y_{0}-x^{n}} d x .
$$

Further details as well as examples demonstrating the reliability of this method are given in Refs. [10, $16]$.

\section{Results and summary}

As an example of our work we consider $p+{ }^{3} \mathrm{H}$ scattering and in Figs. 1 - 4 we show the differential cross section and selected spin observables for elastic, charge-exchange, and transfer reactions. Further examples can be found in Refs. [16, 26-28]. We use several realistic interaction models, i.e., the charge-dependent Bonn potential (CD Bonn) [29], its coupled-channel extension CD Bonn $+\Delta[30]$, and the inside-nonlocal outside-Yukawa (INOY04) potential by Doleschall $[6,31]$. The latter yields nearly the experimental value for the ${ }^{3} \mathrm{He}$ and ${ }^{3} \mathrm{H}$ binding energy without an additional three-nucleon force. The overall description of the available experimental data is reasonably good. 


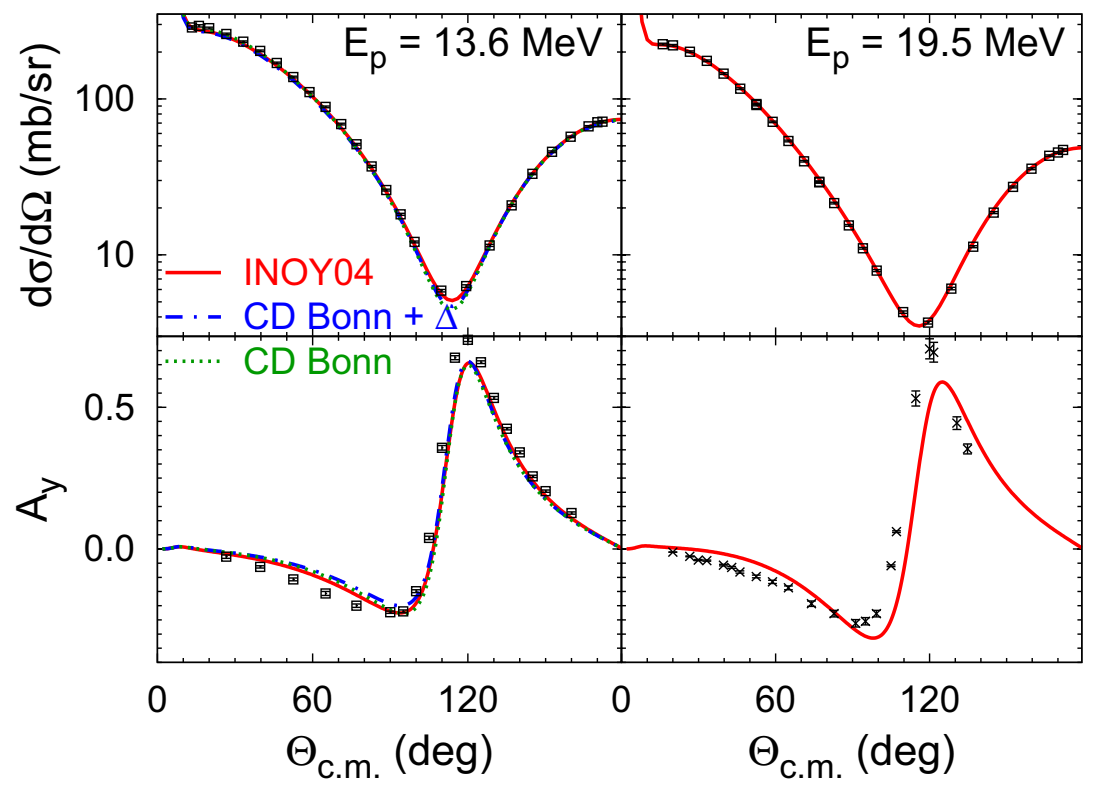

Figure 1. Differential cross section and proton analyzing power of elastic $p-{ }^{3} \mathrm{H}$ scattering at 13.6 and $19.5 \mathrm{MeV}$ proton energy. Results obtained with potentials INOY04 (solid curves), CD Bonn (dotted curves), and CD Bonn $+\Delta$ (dashed-dotted curves) are shown. The data are from Refs. $[17,18]$.

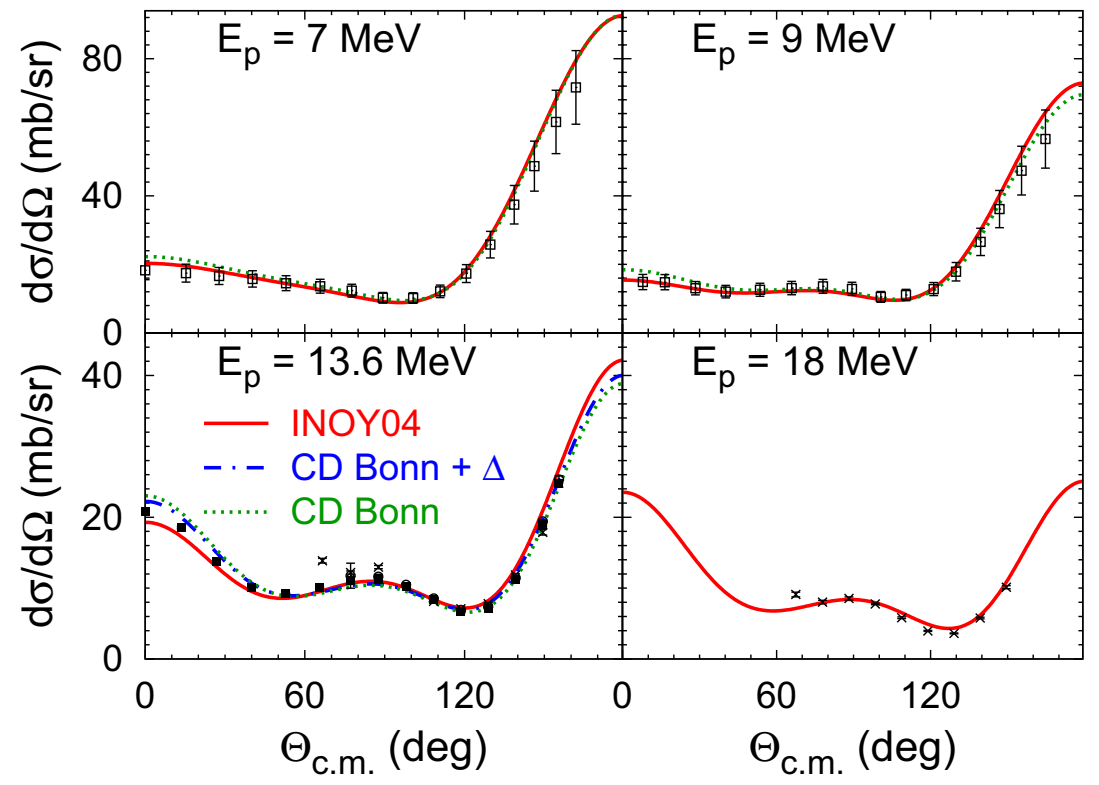

Figure 2. Differential cross section of ${ }^{3} \mathrm{H}(p, n)^{3} \mathrm{He}$ reaction at 7, 9, 13.6, and $18 \mathrm{MeV}$ proton energy. Curves as in Fig. 1. The data are from Refs. [19-22]. 

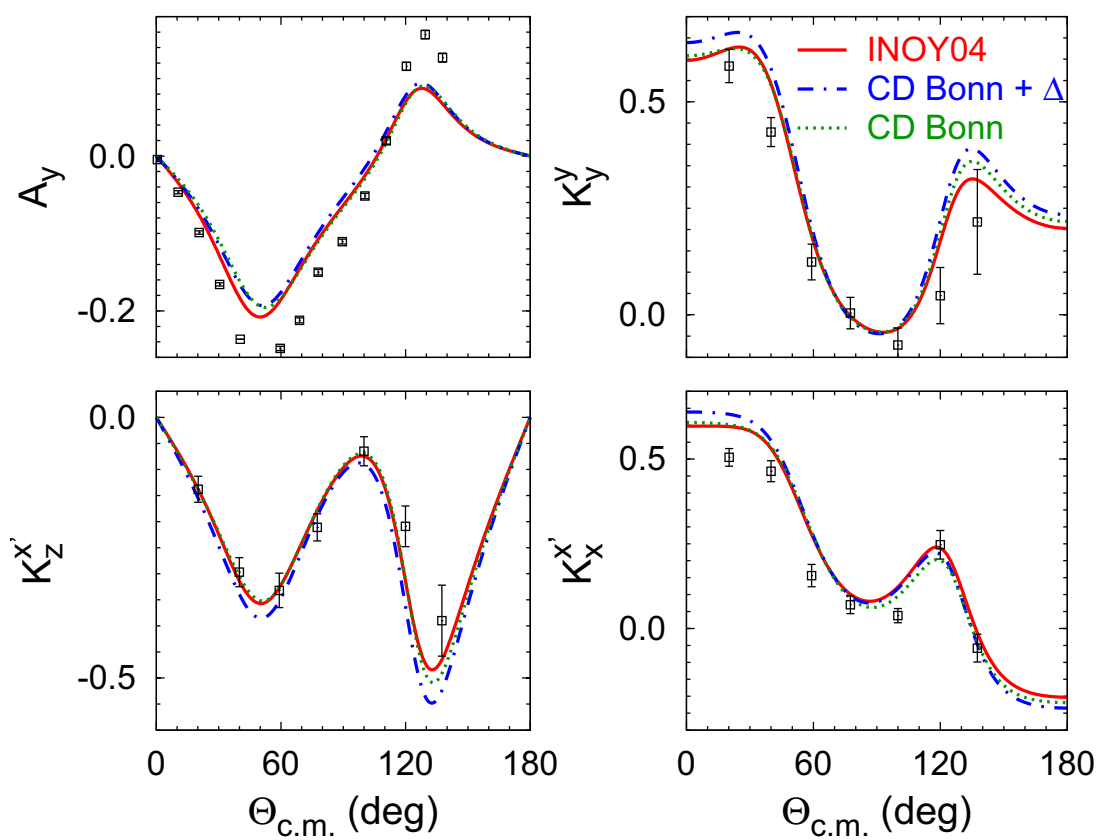

Figure 3. Proton analyzing power and proton to neutron polarization transfer coefficients of ${ }^{3} \mathrm{H}(p, n)^{3} \mathrm{He}$ reaction at $13.6 \mathrm{MeV}$ proton energy. Curves as in Fig. 1. The data are from Refs. [23, 24].

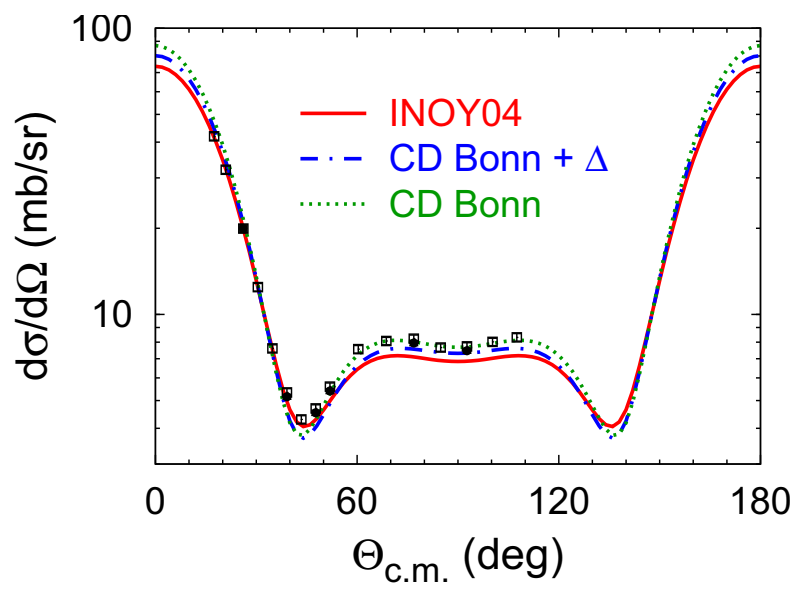

Figure 4. Differential cross section of ${ }^{3} \mathrm{H}(p, d)^{2} \mathrm{H}$ reaction at $13.6 \mathrm{MeV}$ proton lab energy. Curves as in Fig. 1 . The data are from Refs. [17, 25]. 


\section{References}

[1] O.A. Yakubovsky, Yad. Fiz. 5, 1312 (1967) [Sov. J. Nucl. Phys. 5, 937 (1967)]

[2] E.O. Alt, P. Grassberger, W. Sandhas, JINR report E4-6688, 1 (1972)

[3] P. Grassberger, W. Sandhas, Nucl. Phys. B2, 181 (1967)

[4] M. Viviani, A. Kievsky, S. Rosati, E.A. George, L.D. Knutson, Phys. Rev. Lett. 86, 3739 (2001)

[5] A. Kievsky, S. Rosati, M. Viviani, L.E. Marcucci, L. Girlanda, J. Phys. G 35, 063101 (2008)

[6] R. Lazauskas, J. Carbonell, Phys. Rev. C 70, 044002 (2004)

[7] A. Deltuva, A.C. Fonseca, Phys. Rev. C 75, 014005 (2007)

[8] A. Deltuva, A.C. Fonseca, Phys. Rev. Lett. 98, 162502 (2007)

[9] M. Viviani, A. Deltuva, R. Lazauskas, J. Carbonell, A.C. Fonseca, A. Kievsky, L.E. Marcucci, S. Rosati, Phys. Rev. C 84, 054010 (2011)

[10] A. Deltuva, A.C. Fonseca, Phys. Rev. C 86, 011001(R) (2012)

[11] R. Lazauskas, Phys. Rev. C 91, 041001 (2015)

[12] W.H. Press, B.P. Flannery, S.A. Teukolsky, W.T. Vetterling, Numerical Recipes (Cambridge University Press, Cambridge, 1989)

[13] L. Schlessinger, Phys. Rev. 167, 1411 (1968)

[14] F.A. McDonald, J. Nuttall, Phys. Rev. A 4, 1821 (1971)

[15] H. Kamada, Y. Koike, W. Glöckle, Prog. Theor. Phys. 109, 869L (2003)

[16] A. Deltuva, A.C. Fonseca, Phys. Rev. C 91, 034001 (2015)

[17] J.L. Detch, R.L. Hutson, N. Jarmie, J.H. Jett, Phys. Rev. C 4, 52 (1971)

[18] R. Darves-Blanc, N. Sen, J. Arvieux, A. Fiore, J. Gondrand, G. Perrin, Lett. Nuovo Cimento 4, $16(1972)$

[19] W.E. Wilson, R.L. Walter, D.B. Fossan, Nucl. Phys. 27, 421 (1961)

[20] M. Drosg, Nucl. Sci. Eng. 67, 190 (1978)

[21] N. Jarmie, J.H. Jett, Phys. Rev. C 16, 15 (1977)

[22] R.G. Allas, L.A. Beach, R.O. Bondelid, E.M. Diener, E.L. Petersen, J.M. Lambert, P.A. Treado,

I. Slaus, Phys. Rev. C 9, 787 (1974)

[23] J.J. Jarmer, R.C. Haight, J.E. Simmons, J.C. Martin, T.R. Donoghue, Phys. Rev. C 9, 1292 (1974)

[24] R.C. Haight, J.E. Simmons, T.R. Donoghue, Phys. Rev. C 5, 1826 (1972)

[25] N. Jarmie, J.H. Jett, Phys. Rev. C 10, 54 (1974)

[26] A. Deltuva, A.C. Fonseca, Phys. Rev. Lett. 113, 102502 (2014)

[27] A. Deltuva, A.C. Fonseca, Phys. Rev. C. 90, 044002 (2014)

[28] A. Deltuva, A. Fonseca, Phys. Lett. B 742, 285 (2015)

[29] R. Machleidt, Phys. Rev. C 63, 024001 (2001)

[30] A. Deltuva, R. Machleidt, P.U. Sauer, Phys. Rev. C 68, 024005 (2003)

[31] P. Doleschall, Phys. Rev. C 69, 054001 (2004) 